\section{Thin-layer Chromatography of Linear Oligosaccharides}

\author{
Ken-ichi Kanaya, Seiya Chiba \\ and Tokuji SHIMOMURA \\ Department of Agricultural Chemistry, \\ Faculty of Agriculture, Hokkaido \\ University, Sapporo
}

Received April 21, 1978

Thin-layer chromatography (TLC) has been applied to the separation and the identification of oligosaccharides..$^{1 \sim 4)}$ However, there are few solvent systems which give satisfactory separation of oligosaccharides having more than six glucose residues. In a previous paper $^{2)}$ we reported a solvent system of $n$-butanolisopropanol-water (10: 5: 4 by volume), but clear separation was observed only in the case of oligosaccharides consisting of six or less glucose units. Thus, more effective solvent systems for the separation and identification of oligosaccharides having more than six glucose units as well as those with lesser units are still needed.

The present paper deals with the TLC of a series of malto- or isomalto-oligosaccharides by a new solvent system of $n$-butanol-isopropanol-water $(3: 12: 4$ by volume).

Silica gel (Kieselgel G, Type 60, Merck) was spread on glass plates in the usual manner to a thickness of $250 \mu$. After being air-dried, the plates were heated at $110^{\circ} \mathrm{C}$ for $30 \mathrm{~min}$. The amount of each sugar applied on a plate was $5 \mu \mathrm{g}$. Development was carried out twice at $18 \sim 22^{\circ} \mathrm{C}$ by the ascending technique. The time required for each development was about $150 \mathrm{~min}$ at $20^{\circ} \mathrm{C}$. The saccharides were detected with the anisaldehyde reagent. ${ }^{5)}$

Figures 1 and 2 show the thin-layer chromatograms of a series of maltooligosaccharides of up to eight glucose units and a series of isomaltooligosaccharides of up to eight glucose units, respectively. Using this solvent system, these saccharides could be clearly separated from each other by only double developments, while in the previous solvent system four or more times of developments were required for the separation of maltooligosaccharides of up to six or less glucose units. Table I summarizes the true $R f$ values which were calculated from the relationship ${ }^{6)}$ between the apparent $R f$ values and the times of development, and the apparent $R_{G}$ values which denote the relative mobilities against glucose at $20^{\circ} \mathrm{C}$ in this experiment.

When the logarithm of a partition function, log $R f / 1-R f$, was plotted against the degree of polymeriza-

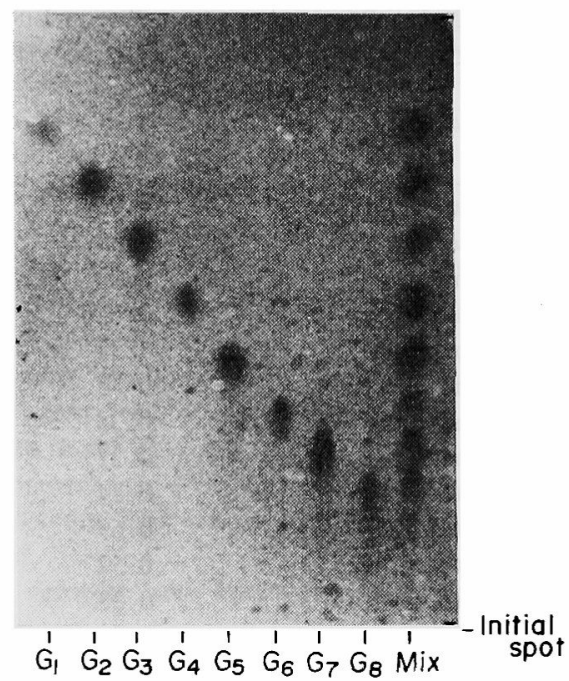

FIG. 1. Thin-layer Chromatogram of Maltooligosaccharides Series.

Chromatoplate, Kieselgel $\mathrm{G}$; solvent system, $n$ butanol-isopropanol-water $(3: 12: 4, \mathrm{v} / \mathrm{v})$; development, ascending technique at room temperature (twice); detection, anisaldehyde reagent.

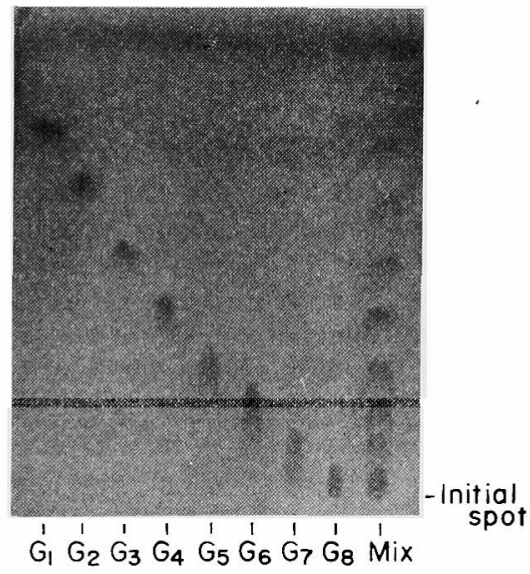

FIG. 2. Thin-layer Chromatogram of Isomaltooligosaccharides Series.

The conditions were the same as described for Fig. 1.

tion, a straight line characteristic for malto- or isomaltooligosaccharide series was obtained in this TLC; such a relationship has also been observed on paper chromatograms. $^{7}$

\section{REFERENCES}

1) C. E. Weill and P. Hanke, Anal. Chem., 34, 1736 (1962). 
Table 1. $R f$ and Apparent $R_{G}$ for Linear HoMOGENEOUS OLIGOSACCHARIDES WITH Double Development

\begin{tabular}{lll}
\hline \multicolumn{1}{c}{ Sugar } & $R_{\mathrm{G}}^{\mathrm{a} p \mathrm{p} .}$ & $R f$ \\
\hline Glucose & 1.00 & 0.52 \\
Maltose & 0.89 & 0.44 \\
Maltotriose & 0.78 & 0.37 \\
Maltotetraose & 0.65 & 0.30 \\
Maltopentaose & 0.53 & 0.23 \\
Maltohexaose & 0.43 & 0.18 \\
Maltoheptaose & 0.35 & 0.15 \\
Maltooctaose & 0.27 & 0.11 \\
Isomaltose & 0.84 & 0.41 \\
Isomaltotriose & 0.69 & 0.31 \\
Isomaltotetraose & 0.52 & 0.23 \\
Isomaltopentaose & 0.38 & 0.16 \\
Isomaltohexaose & 0.26 & 0.11 \\
Isomaltoheptaose & 0.16 & 0.07 \\
Isomaltooctaose & 0.04 & 0.03 \\
\hline
\end{tabular}

2) S. Chiba and T. Shimomura, Agric. Biol. Chem., 29, 486 (1965); idem, ibid., 31, 255 (1967).

3) C. N. Huber, H. D. Scobell, H. Tai and E.E. Fisher, Anal. Chem., 40, 207 (1968).

4) J. C. Schannon and R. G. Creech, J. Chromatogr., 44, 307 (1969).

5) E. Stahl, "Dünnschicht-Chromatograhie," Springer-Verlag, 1962, p. 514.

6) J. A. Thoma, Anal. Chem., 35, 214 (1963).

7) D. French and G. M. Wild, J. Am. Chem. Soc., 75, 2612 (1953). 